\title{
Reflexiones interculturales en la obra de Bernat Metge: una revisión sociocultural de un autor «al margen de la ley»
}

\author{
Intercultural Reflections on the Literary Work of Bernat Metge: A \\ Sociocultural Revision of an Author «outside the law»
}

\author{
Òscar O. SANTOS-SOPENA \\ oscarsantossopena@gmail.com
}

Universidad Politécnica de Madrid

\begin{abstract}
Resumen: El pensador barcelonés Bernat Metge (1340/46-1413) es uno de los autores clave de la literatura catalana como evidencian las numerosas investigaciones llevadas a cabo desde el siglo XIX. En esta investigación partimos de un Metge controvertido y «al margen de la ley» que escribe obras como Llibre de Fortuna e Prudència (1381) y Lo somni (1399) que a través de la palabra, la ironía y el humor desarrolla narrativamente sus problemas políticos y sirven para probar su inocencia en los procesos abiertos en su contra mientras que a su vez plantea algunos de los problemas filosóficos propios del Humanismo europeo (Batllori 1995; Butiná 2007; Gómez Moreno 2011). Este estudio recalca el uso del diálogo satírico humanista que ayuda a entender y adelantar el origen de la modernidad y la existencia de un pensamiento humanista en las letras catalanas.
\end{abstract}

Palabras clave: Interculturalidad, Humanismo, Bernat Metge, autobiografía

Abstract: As evidenced by the numerous researches carried out since the 19th Century the Catalan writer Bernat Metge (1340 / 46-1413) is one of the key authors of Catalan literature. In this research, we understand Metge as controversial writer, an «outside the law» author who writes works such as Llibre de Fortuna e Prudència (1381) and Lo somni (1399) that through words, irony, and humor narratively develops their political issues and it helps to prove his innocence in some of the open penal processes against him, while at the same time raising some of the philosophical problems of European Humanism (Batllori 1995; Butiná 2007; Gómez Moreno 2011). This study highlights the use of satirical humanist dialogue that helps to understand and promote the origin of modernity and the existence of humanistic thought in Catalan literature.

Keywords: Interculturality, Humanism, Bernat Metge, autobiography

DATA PRESENTACIÓ: 15/11/2020 ACCEPTACIÓ: 20/11/2020 • PUBLICACIÓ: 10/12/2020

SCRIPTA, Revista internacional de literatura i cultura medieval i moderna, núm. 16 / desembre 2020 / pp. 14-23 ISSN: $2340-4841 \cdot$ doi:10.7203/SCRIPTA.16.19212 
Òscar O. Santos-Sopena. Reflexiones interculturales en la obra de Bernat Metge: una revisión sociocultural de un autor «al margen de la ley»

\section{Introducción: Bernat Metge y las primeras reflexiones humanistas}

El escritor barcelonés Bernat Metge (1340/46-1413) es uno de los autores clave en la introducción de la corriente humanista en la literatura catalana como evidencian las numerosas investigaciones llevadas a cabo desde el siglo XIX (Batllori 1995; Butiná 2007; Gómez Moreno 2011). En esta investigación partimos de un Metge controvertido y «al margen de la ley» que escribe obras como Llibre de Fortuna e Prudència (1381) y Lo somni (1399) que a través de la palabra, la ironía y el humor desarrolla narrativamente sus encontronazos políticos y que sirven para probar su inocencia en los procesos abiertos en su contra mientras que a su vez plantea algunos de los problemas filosóficos propios del Humanismo europeo (Batllori 1995). Este estudio recalca la conexión vida-texto del pensador barcelonés que ayuda a entender y adelantar el origen de la modernidad en la Península ibérica y, que además plantea la existencia de un pensamiento humanista, intercultural y de origen cristiano en las letras catalanas.

Bernat Metge, como primer autor con un sentido humanista en la Península ibérica, procura los elementos suficientes para entender bien cómo se configura inicialmente el pensamiento minucioso del Humanismo y cómo se transfiere en sus obras: un autor «al margen de ley» (desde un punto de vista figurativo y metafórico, aunque a veces real si pensamos en su experiencia de vida) como veremos en algunos ejemplos biográficos y argumentativos que hemos seleccionado para este estudio.

Metge es un claro ejemplo de la convergencia de la tradición grecolatina y cristiana, elemento esencial en los autores del Humanismo mediterráneo. Además, como sucede en los humanistas italianos, Metge presenta de forma más difusa dicha esencia humanista europea ya que converge con una amplia tradición cristiana ya existente en el ecosistema sociocultural de la Península ibérica y en sus tradiciones literarias. De ahí que se vaya a analizar profusamente a un Metge, cuyo estilo asume estas dos tradiciones, a pesar de que, aunque pionero y revolucionario, no llega a influir de manera directa e inmediata posteriormente en las letras peninsulares. Esta cuestión no desmerece la importancia de su producción y sobre todo la tradición que recoge y que es un claro reflejo de la mirada intercultural propia del Humanismo cristiano.

Para un análisis de carácter intercultural de este autor es necesario una triple metodología: la comparatista, la cultural y la analítica. Gracias a este triple prisma se establece un polisistema cultural de convivencia-intercambio literario-cultural que viene reflejado en los acontecimientos vitales del propio autor y ver de qué manera se ve reflejado en su obra-texto. Por lo tanto, los objetivos que se plantean este este trabajo es:

1. Profundizar en las relaciones interdisciplinarias y sus mecanismos y manifestaciones interculturales y ver de qué manera se ven reflejados en la obra de un autor como Bernat Metge;

2. Establecer una cronología historiográfica determinada, en la que se nos ofrece (re)pensar ciertos procesos teóricos y categorías dentro de la periodización histórica;

SCRIPTA, Revista internacional de literatura i cultura medieval i moderna, núm. 16 / desembre 2020 / pp. 14-23 ISSN: 2340-4841 · doi:10.7203/SCRIPTA.16.19212 
Òscar O. Santos-Sopena. Reflexiones interculturales en la obra de Bernat Metge: una revisión sociocultural de un autor «al margen de la ley»

3. Examinar el contexto político-social presente en la producción textual de un autor como Bernat Metge; y finalmente

4. Reflexionar en torno a las relaciones literarias-culturales como mecanismo de (de)construcción histórica.

Estos objetivos nos llevan a recalar la figura de Metge como autor humanista y a resaltar el valor de su pensamiento humanista cristiano y reinterpretar su obra desde una vertiente intercultural.

\section{Componente humanista e intercultural en Bernat Metge}

Hay nociones que se deben tener en cuenta para entender la figura de Bernat Metge y el «perfecto humanista». La primera es que parte del conocimiento del cristianismo, elemento que sí es común en toda la Europa mediterránea, aunque de diferente intensidad como se ha comentado con anterioridad. Por lo tanto, ser humanista es ser un escritor universitario o eclesiástico, político, profesor, filósofo o funcionario que hace patente, en su vida intelectual, un interés profundo por la tradición clásica. Un individuo reflexivo que presta atención al contenido filosófico del pensamiento clásico y cristiano. Un autor que ya en la Edad Media comienza a establecer un vínculo entre reflexión y crítica, emociones y sentimientos, sociedad, ley, mercado y organización económica.

En segundo lugar, para Batllori el Humanismo peninsular primero es helenista y luego latinista, además de tener un claro recorrido geográfico (Batllori 1995). Aparece en la Península ibérica con el monarca aragonés Pere III «el Cerimoniós» (1336-1387) que creó un cuerpo de traductores dentro de la Cancelleria Reial (Cancillería Real) en la Corona de Aragón, referencia para la normalización lingüística, de la lengua catalana por medio del lenguaje escrito con la que el cuerpo de funcionarios reales redactaba los documentos. Por lo tanto, su inicio está enmarcado dentro de la esfera política y legislativa, si bien una de las características primordiales de esta concepción humanística es esa tendencia a lo práctico de estos textos administrativos-gubernamentales, y por tanto de sus autores. Sin embargo, éstos no dejan de lado su parcela creativa entrelazada con la concepción de la traducción y edición -entendida como recuperación cultural de los clásicos grecolatinos. Esta recuperación cultural nace de este encuentro comparatista y de relaciones interculturales que se está trazando en esta reflexión.

Por último, el conocimiento e intersección cultural de Bernat Metge es un ejemplo claro de su hacer y sentido humanista. Metge parte de la tradición anterior y un referente claro es el autor medieval Ramón Llull (1232/33-1315/16) y su misticismo representado en sus obras:

En buen número de obras de Ramón Llull se hace manifiesto el misticismo del gran escritor, que ya aparece con intensidad y con sus características más propias en el Libre de contemplació, pero que se sintetiza y se desarrolla admirablemente en el Libre d'Amic e Amat e culmina en el Arbre de filosofia d'amor. (Riquer 1972: 35) 
Òscar O. Santos-Sopena. Reflexiones interculturales en la obra de Bernat Metge: una revisión sociocultural de un autor «al margen de la ley»

Autor entendido como un prehumanista, además de pensador, teólogo y filósofo mallorquín que recoge y entiende a la perfección la globalidad cultural del contexto mediterráneo y europeo de su época. Siendo este el componente intercultural al que nos referimos a la hora de entender la vía de la razón como forma de explicación. Llull y Metge juegan con la razón y ambos conocen sus límites. Es ahí donde el esfuerzo racional para llegar a creer es común en ambos y es motivo literario. Esta cuestión se traduce en Metge en la concepción del Humanismo cristiano.

\section{Análisis: obra como reflejo vital}

Bernat Metge promueve a través de sus obras (sobre todo las que hemos seleccionado) un proceso reivindicativo de hombre con un vaivén «al margen de la ley» y que mucho tiene que ver con sus acontecimientos vitales. Esta cuestión tiene mucho que ver con el carácter y esencia metginana. Por ello, los textos tienen a su vez cierto carácter precursor ya que se verán reflejados los aspectos que reemprende y aquellos que desdibuja en su ecosistema vital.

Metge, en los albores del Humanismo peninsular, gracias a su humor e innovación lingüística y temática. Así pues, en 1381 con su Llibre de Fortuna e Prudència, obra compuesta por 1194 versos octosílabos y escrita en primera persona, llega el momento de este preludio alegórico humanístico, en este caso a partir de la creación de un mundo fabuloso-fantástico que ayuda a exponer cuestiones de carácter filosófico. Una cuestión interesante por señalar es que, aunque el uso de la primera persona está claro, no conlleva, gracias a su empleo del lenguaje, la identificación del autor con el protagonista. Esta cuestión es interesante a la hora de señalar el uso de sus textos a la posible defensa, o no defensa, de lo acontecido es su escena vital.

En esta obra encontramos los esquejes del pensamiento y metodología humanista, que produce una mirada global, para explicar su viaje a la isla de la Fortuna. Todos los acontecimientos que relata el barcelonés sirven para probar su inocencia en los primeros procesos abiertos en su contra. La virtud y la inocencia se convierten en aspectos claves en su planteamiento temático mientras que a su vez plantea algunos de los problemas filosóficos que luego continuará. En esta obra tenemos referencias específicas desde el comienzo del texto de vida y se convierte la defensa de su inocencia en punto de partida a este concepto de defensa, o no defensa, a través de la escritura. Elemento esencial para tener en cuenta y que estamos desarrollando en este y otros trabajos previos, y es aquí donde nace esta reflexión. ${ }^{1}$

1 El estudio de este viaje cultural e ideológico lo vengo trazando desde los inicios de mi publicación e investigación académica. Por ello, es necesario hacer constancia de la continuación que supone esta tesis doctoral hacia estos trabajos publicados con antelación y que sirven de referencia y punto de partida, especialmente en este capítulo:

Santos-Sopena, Òscar O. Review: «Os Sonhos na História.» Ed. Ricardo da Costa. Alicante/Madrid: e-Editorial IVITRA Políglota. Estudis, Edicions i Traduccions / Atenea, 2014. Publisher: Mirabilia; Electronic Review of Antiquity \& Middle Ages (UFES - Brasil) and Journal of Institut d'Estudis Medievals - Universitat Autònoma de Barcelona (UAB - España). Barcelona: Institut d'Estudis Medievals - UAB (2015): 180-198. Web. 20 Sept. 2015. 
Òscar O. Santos-Sopena. Reflexiones interculturales en la obra de Bernat Metge: una revisión sociocultural de un autor «al margen de la ley»

Además, su vida condiciona y genera literariamente hablando un diálogo onírico (Santos-Sopena 2013), por eso es necesario hacer mención, aunque de forma sucinta, a algunos aspectos de su biografía. Bernat Metge nace en Barcelona en el seno de una familia que se encargaba de suministrar especias en la vecina corte barcelonesa. Su madre quedó viuda muy pronto y se volvió a casar con Ferrer Sayol protonotario de la mujer del rey Pere III «el Cerimoniós», Elionor de Sicilia. La nueva figura paterna fue la encargada en introducirle el interés y el gusto por la temática grecolatina.

Es interesante observar que siguiendo la senda de su padrastro va a entrar dentro del cuerpo de funcionarios reales. En 1371 fue nombrado ayudante de registro en la Cancillería de la reina Elionor. Es con este cargo que comienza su carrera funcionarial-institucional: primero como ayudante del registro, luego como escribano del rey Joan I, y posteriormente, en 1390, como secretario real del mismo monarca y de la reina Violant de Bar. Esta situación privilegiada le creó muchas enemistades, que se transformaron en acusaciones de malversación de dinero público que marcarán su futuro profesional. Se sabe que en esta época fue procesado, pero desconocemos qué denuncias concretas le imputaban en esta primera acusación (Badia 1999: 9-13).

\footnotetext{
- «Soñadores Literarios: una (re)lectura de Los sueños de Quevedo a través del humanismo peninsular.» Actas VI Congreso de la Asociación Hispánica de Humanidades (AHH): Literatura y cine: el Bicentenario de la Independencia Iberoamericana y de la Constitución de Cádiz. Eds. Jorge H. Valdivieso y Enrique Ruiz-Fornells. Turlock, CA: Editorial Orbis Press, Ensayos / Serie Reflexión, V. 16, 2014. 162-170.

- Soñadores Literarios: De Bernat Metge a Francisco de Quevedo. «El Sueño» y su aportación al relato histórico-cultural de dos épocas. Tesis Doctoral. College Park, MD: U. of Maryland, 2013a.

—. «Descubriendo técnicas y motivos narrativos en Lo somni de Bernat Metge.» Bernat Metge humanista i poliglota. eHumanista/IVTTRA. Eds. Julia Butiñá Jiménez, Antonio Cortijo-Ocaña y Vicent Martines Peres. Literature, Language and Culture of the Crown of Aragon/ Literatura, Llengua i Cultura de la Corona d'Aragó 4 (2013b): 35-148. Web. 22 Sept. 2015.

—. «Diálogo y encuentro cultural Mediterráneo: el Humanismo de Bernat Metge.» Escribir y persistir. Estudios sobre literatura en catalán de la Edad Media a la Renaixença (3 vols.). Ed. Vicent Josep Escartí. Buenos Aires y Los Ángeles: Argus-a. Arts \& Humanities, 2013c. 40-59.

- Review: «L’Humanisme a la Corona d'Aragó.» Eds. Julia Butiñá Jiménez y Antonio Cortijo-Ocaña. eHumanistal IVITRA 1 (2012). Publisher: Mirabilia. Electronic Review of Antiquity \& Middle Ages (UFES - Brasil) and Journal of Institut d'Estudis Medievals - Universitat Autònoma de Barcelona (UAB - España) 15 (2012a): 384-391. Web. 14 Jul. 2015.

- Review: «Metge, Bernat. El Llibre de Fortuna e Prudència. Ed. Miquel Marco. Barcelona: Reial Academia de Bones Lletres-IVITRA (Prometeo), 2010.» Publisher: Bolletí de la Real Academia de les Bones Lletres de Barcelona - RABLB, 2012b.

- «Llibre de l'Orde de Cavalleria. Libro de la orden de caballería. The book of the Order of Chivalry.» Los mundos de Ramón Llull en las lenguas de hoy. Ed. Julia Butiñá Jiménez. Madrid: UNED, 2012c. 32-44.

—. Review: «Llibre de Fortuna i Prudència: un Bernat Metge humanista.» Metge, Bernat. El Llibre de Fortuna e Prudència. Ed. Miquel Marco. Barcelona: Reial Academia de Bones Lletres-IVITRA (Prometeo), 2010. eHumanista (2011a). Web. 28 Feb. 2013.

- «Hombres, hombres y más hombres: creando la figura del caballero español desde la Edad Media hasta fines del Barroco.» Actas Seleccionadas del Congreso Internacional del ALDEEU. Eds. Alicia de Gregorio y María José Luján. Alcalá de Henares: ALDEEU-Spanish Professionals in America, Inc., 2011b. 249-267.
}

SCRIPTA, Revista internacional de literatura i cultura medieval i moderna, núm. 16 / desembre 2020 / pp. 14-23 ISSN: 2340-4841 · doi:10.7203/SCRIPTA.16.19212 
Òscar O. Santos-Sopena. Reflexiones interculturales en la obra de Bernat Metge: una revisión sociocultural de un autor «al margen de la ley»

Posteriormente le siguieron otros cargos en la administración pública que le hicieron crecer dentro del cuerpo diplomático catalano-aragonés. Muchas de estas responsabilidades son significativas para entender su papel crucial para las letras peninsulares. Por ejemplo, durante el reinado de Joan I, Metge actuó de embajador, entre febrero-abril de 1395 fue enviado a la corte pontificia de Aviñón. Este periodo en la erudita-controvertida corte papal fue fundamental para su formación y crecimiento intelectual por su encuentro con la tradición humanística italiana-mediterránea. Esta situación le permitió consultar códices de Petrarca.

En 1396, al cabo de dos meses de la muerte repentina del rey Joan I, comenzó un proceso por corrupción y traición, iniciado por la esposa del nuevo monarca María de Luna, contra 38 consejeros reales, entre los que se encontraba Bernat Metge, estaban acusados presuntamente de haber aconsejado mal al rey, de haber aceptado sobornos, y se les consideraba culpables de la muerte repentina del monarca (Tavani 1998: 11-12). En resumen, Metge fue procesado por traición y corrupción ya que la muerte del monarca se había convertido en un tema problemático y razón de estado para la opinión pública, ya que se creía que el alma del rey no había podido recibir la confesión ni los auxilios necesarios a causa de una muerte tan repentina (Badia 1999: 16). No sólo eso, también se especulaba sobre la condena y la no salvación del alma del rey, cuestión que suponía un problema moral y teológico en el contexto cristiano de la época.

Metge nos lleva a lo largo de cuatro libros o tratados por una defensa de la inmortalidad del alma, una discusión sobre la esencia de la religión y sobre el sentido de la dignidad y entidad moral del ser humano. Metge va dialogando con el alma del rey fallecido sobre la inmortalidad del alma, con Tiresias y Orfeo sobre el ineludible destino del alma hacia la salvación, y con Tiresias de nuevo para realzar la entidad moral de la mujer. Por ejemplo, en el Libro I, como indica Tavani, el tema esencial de esta obra es «captar per ell mateix el perdó del nou rei, aconseguir la rehabilitació i la restitució dels càrrecs administratius que li havien tret $\mathrm{i}$ que abans li havien permès de viure sense problemas econònomics» (1998: 19-22).

Concretamente, este proceso duró hasta 1398, y el sucesor de Joan I, su hermano Martí I «l'Humà», absolvió a todos los encausados, después de que Metge presuntamente estuviera aproximadamente dos años en prisión. En este punto es necesario destacar que no es seguro ni que estuviera en prisión Bernat Metge, lo que sí sabemos es que sus amigos sí. Aunque la cuestión de la cárcel no es segura, no está demostrada completamente. Batllori, quien incluso da la cifra de dos años de su estancia en prisión (1995: 47), donde gestaría la obra, insistía en que no se puede asegurar que lo llegase a estar. En cuanto a la documentación de estos hechos, Riquer (1959: 215-244) la recoge profusamente (da relación de 177 documentos). Se ha tratado más el tema, sobre todo en la edición de Cingolani (edición de 2006), que vuelve a dar mucho relieve a estos hechos, pero la prisión sigue sin documentarse. Por eso, han planteado algunos autores que se pueda considerar como un motivo literario. 
Òscar O. Santos-Sopena. Reflexiones interculturales en la obra de Bernat Metge: una revisión sociocultural de un autor «al margen de la ley»

Así pues, después de la muerte de Joan I (1396), la situación privilegiada de Metge en la corte queda truncada por este inesperado acontecimiento. Comienza a escribir Lo somni, por eso la obra ha sido interpretada por la crítica como una defensa a sus imputaciones de los cargos de malversación de fondos y muerte del monarca. Metge gracias a su interés por la tradición clásica, transforma esta apología en una obra maestra, que presenta un discurso y un debate que sirve como estrategia legalretórica contra dichas acusaciones. La obra tiene como objetivo conseguir nuevamente la confianza y la posición social de la que gozó Metge antes de la coronación del monarca Martí I «l'Humà» o «l'Eclesiàstic», que reinó del 1396-1410 (Tavani 1998 :12).

Después de la resolución del conflicto, Bernat Metge pasa al servicio del nuevo soberano hasta su muerte. Tras la defunción del rey y la llegada de la dinastía castellana de los Trastámara [en Castilla (1369-1555), en Aragón (1412-1555), en Navarra (1425-1479) y en Nápoles (1458-1501 y 1504-1555)]328 ya no asumió más cargos oficiales. Es decir, Metge reivindicó su posición en la corte, para luego no ocupar ningún cargo más administrativo y morir en la Barcelona de 1413 (Badia 1999: 17).

\section{A modo de conclusión: reflexiones socioculturales e interculturales en Metge}

Lo somni pretende como indicábamos anteriormente captar el perdón del nuevo monarca catalano-aragonés y así restituir los cargos políticos del propio Bernat Metge, y con este texto lo consigue (Tavani 1998: 19). Además, siempre desde una perspectiva irónica intrínseca en el texto y dicho componente queda reflejado en los 4 libros que lo componen, aunque con diferencias significativas (Santos-Sopena 2013). Así, Lo somni es una manera indirecta y justificada de vincular los contenidos filosófico-morales propios del cristianismo con los acontecimientos sociopolíticos y, además, tienen un amplio elemento pedagógico por acercarse a estas dos tradiciones, como sucede también en Juan Luis Vives (Calero 2015). No cabe la menor duda de que después de una lectura cuidadosa del texto nos encontramos con subtextos y subcategorías clásicas y cristianas que conforman el Humanismo cristiano de Metge, que sufre una profunda dependencia en torno al componente didáctico tan propio de la tradición peninsular. Precisamente ese componente didáctico lo conectamos con el componente intercultural y ofrece una mirada de encuentro. Metge es precisamente eso, una reminiscencia del encuentro intercultural a través de su forma de entender la tradición grecolatina y cristiana.

Lo somni es por lo tanto un ensayo didáctico de lenguaje lírico y dramático. Sin duda, a raíz de este texto se consolida la mirada innovadora humanística de Bernat Metge directamente continuadora del Humanismo italiano y que aúna la tradición mediterránea. Siendo estas una de las conclusiones esenciales que se pueden observar tras revisar su manera de exponer sus acontecimientos autobiográficos. Por lo tanto, se convierte en punto de encuentro entre la escolástica medieval y la corriente humanista petrarquista en que Metge se convierte en uno de los máximos escritores 
Òscar O. Santos-Sopena. Reflexiones interculturales en la obra de Bernat Metge: una revisión sociocultural de un autor «al margen de la ley»

peninsulares, aunque no por ello más conocido y celebrado posteriormente, siendo autor fundamental gracias a su papel de introducción del estilo humanista cristiano en la literatura catalana y en el Humanismo hispánico.

Un personaje que quizás «al margen de la ley» y de los procesos creativos y artísticos de la época, o tal vez no tanto pero que sí ofrece y representa la llegada de la modernidad occidentalmediterránea en la Península ibérica en general y en las letras catalanas en particular. Por lo tanto, esa interculturalidad se ve reflejada en su conciencia creativa e intelectual y que se traduce en el pensamiento panmediterráneo. Una experiencia vital la de Bernat Metge que seguirá ofreciendo nuevas vías de entendimiento y recogimiento académico. 
Òscar O. Santos-Sopena. Reflexiones interculturales en la obra de Bernat Metge: una revisión sociocultural de un autor «al margen de la ley»

\section{Bibliografía}

Areces, José Ramón (2011). «Lo somni o la reivindicació ontològica de l’home,» en Butinyà, J. \& A. Cortijo Ocaña (eds), L'Humanisme a la Corona d'Aragó (en el context hispànic i europeu), Potomac, MD, Scripta Humanistica, pp. 79-100.

Badia, Lola (1999). Lo somni. Autor Bernat Metge. Barcelona, Quaderns Crema.

Batllori, Miquel (1995). Obra completa. V. De l'Humanisme i del Renaixement. Valencia, Tres i Quatre.

Butiñá Jiménez, Julia (1995) «El diálogo de Bernat Metge con Ramón Llull: dos nuevas fuentes tras Lo somni.» Medioevo y literatura, I-IV. Granada, U. de Granada

_- (2003) «Unes notes sobre Metge, Llull i Juvenal.» Homenatge al professor Miquel Batllori, 4, Randa 51, pp. 7-29.

- (2005) «El diálogo en Llull y en Metge.» Estudios Hispánicos 12, Miscelánea de Literatura española y comparada. Homenaje a Roberto Mansberger Amorós, coord. por A. August Zarebska, J. Butiñá, J. Ziarkovska, U. de Wroclaw, pp. 107-120.

- (2007) «¿Una muestra de la unidad de pensamiento luliano en un humanista del siglo XIV? (Avanzando en la interpretación de Lo somni de Bernat Metge).» Studia Pbilologica Valentina 10.7, pp. 65-94.

. (2014). «Entre 2013 i 2016: entre Metge i Llull.» Homenatge a Carles Miralles. Vol. II. Barcelona, U. de Barcelona, pp. 51-69.

Calero, Francisco (2012). «La nueva imagen de Luis Vives.»eHumanista/IVTTRA 1, pp. 15-30. Web. 13 Jul. 2015.

Gómez Moreno, Ángel (1994) España y la Italia de los humanistas: primeros ecos. Madrid, Gredos.

- (2008) Claves Hagiográficas de la literatura española: del Cantar de Mio Cid a Cervantes. Madrid, Iberoamericana.

—_. (2010) «Del Duecento al Quattrocento: Italia en España, España en Italia.» Entre Italia y España-Ínsula, 757-758, pp. 7-11.

(2011). «Renaixement i Humanisme a Espanya: esculls, principis vertebradors i dades històriques,» en Butinyà, J. \& A. Cortijo Ocaña (eds), L'Humanisme a la Corona d'A ragó (en el context hispànic i europeu), Potomac, MD, Scripta Humanistica, pp. 229-262.

Riquer, Martí de (1947) Resumen de literatura catalana. Barcelona, Seix Barral.

—_. (1959) Lo somni. Obras de Bernat Metge. Trad. y estudio de M. de Riquer. Barcelona, U. de Barcelona.

—. (1972) Literatura catalana medieval. Barcelona, Ayuntamiento de Barcelona - Delegación de Servicios de Cultura.

Riquer, Martí de, y Antoni Comas (1964) Història de la literatura catalana. Vol. I. Barcelona, Ariel. 
Òscar O. Santos-Sopena. Reflexiones interculturales en la obra de Bernat Metge: una revisión sociocultural de un autor «al margen de la ley»

Santos-Sopena, Òscar O. (2011a) Review: «Llibre de Fortuna i Prudència: un Bernat Metge humanista.» Metge, Bernat. El Llibre de Fortuna e Prudència. Ed. Miquel Marco. Barcelona, Reial Academia de Bones Lletres-IVITRA (Prometeo), eHumanista. Web. 28 Feb. 2013.

— - (2011b) «Hombres, hombres y más hombres: creando la figura del caballero español desde la Edad Media hasta fines del Barroco.» Actas Seleccionadas del Congreso Internacional del ALDEEU. Eds. Alicia de Gregorio y María José Luján. Alcalá de Henares: ALDEEU-Spanish Professionals in America, Inc., pp. 249-267.

—. (2012a) Review: «L’Humanisme a la Corona d'Aragó.» Eds. Julia Butiñá Jiménez y Antonio Cortijo-Ocaña. eHumanista/IVITRA 1 (2012). Publisher: Mirabilia. Electronic Review of Antiquity \& Middle Ages (UFES - Brasil) and Journal of Institut d'Estudis Medievals Universitat Autònoma de Barcelona (UAB - España) 15, pp. 384-391. Web. 14 Jul. 2015.

. (2012b) Review: «Metge, Bernat. El Llibre de Fortuna e Prudència. Ed. Miquel Marco. Barcelona: Reial Academia de Bones Lletres-IVITRA (Prometeo), 2010.» Publisher: Bolletí de la Real Academia de les Bones Lletres de Barcelona - RABLB.

- . (2012c) «Llibre de l'Orde de Cavalleria. Libro de la orden de caballería. The book of the Order of Chivalry.» Los mundos de Ramón L bull en las lenguas de hoy. Ed. Julia Butiñá Jiménez. Madrid: UNED, pp. 32-44.

- (2013a) Soñadores Literarios: De Bernat Metge a Francisco de Quevedo. «El Sueño» y su aportación al relato bistórico-cultural de dos épocas. Tesis Doctoral. College Park, MD, U. of Maryland, 2013a.

_- (2013b) «Descubriendo técnicas y motivos narrativos en Lo somni de Bernat Metge.» Bernat Metge humanista i poliglota. eHumanista / IVITRA. Eds. Julia Butiñá Jiménez, Antonio CortijoOcaña y Vicent Martines Peres. Literature, Language and Culture of the Crown of Aragon/ Literatura, Llengua i Cultura de la Corona d'Aragó 4, pp. 35-148. Web. 22 Sept. 2015.

- - (2013c) «Diálogo y encuentro cultural Mediterráneo: el Humanismo de Bernat Metge.» Escribir y persistir. Estudios sobre literatura en catalán de la Edad Media a la Renaixença (3 vols.). Ed. Vicent Josep Escartí. Buenos Aires y Los Ángeles, Argus-a. Arts \& Humanities, pp. 40-59.

- (2014) «Soñadores Literarios: una (re)lectura de Los sueños de Quevedo a través del humanismo peninsular.» Actas VI Congreso de la Asociación Hispánica de Humanidades (AHH): Literatura y cine: el Bicentenario de la Independencia Iberoamericana y de la Constitución de Cádiz. Eds. Jorge H. Valdivieso y Enrique Ruiz-Fornells. Turlock, CA, Editorial Orbis Press, Ensayos / Serie Reflexión, V. 16, pp. 162-170.

- (2015) Review: «Os Sonhos na História.»Ed. Ricardo da Costa. Alicante/Madrid: e-Editorial IVITRA Políglota. Estudis, Edicions iTraduccions / Atenea, 2014. Publisher: Mirabilia;Electronic Review of Antiquity \& Middle Ages (UFES - Brasil) and Journal of Institut d'Estudis Medievals - Universitat Autònoma de Barcelona (UAB - España). Barcelona, Institut d'Estudis Medievals - UAB, pp. 180-198. Web. 20 Sept. 2015.

Tavani, Giuseppe (1998) Lo somni. Ed. Marta Jordà, prólogo de Giuseppe Tavani obra de Bernat Metge. Barcelona, Edicions 62.

SCRIPTA, Revista internacional de literatura i cultura medieval i moderna, núm. 16 / desembre 2020 / pp. 14-23 ISSN: 2340-4841 · doi:10.7203/SCRIPTA.16.19212 